Review

\title{
Sirt3, Mitochondrial ROS, Ageing, and Carcinogenesis
}

\author{
Seong-Hoon Park ${ }^{1}$, Ozkan Ozden ${ }^{1}$, Haiyan Jiang ${ }^{1}$, Yong I. Cha ${ }^{1}$, J. Daniel Pennington ${ }^{1}$, \\ Nukhet Aykin-Burns ${ }^{2}$, Douglas R. Spitz ${ }^{2}$, David Gius ${ }^{1}$ and Hyun-Seok Kim ${ }^{1, *}$ \\ 1 Department of Cancer Biology, Pediatrics, and Radiation Oncology, \\ Vanderbilt University Medical Center, Nashville, TN 37232, USA; \\ E-Mails: romad96@gmail.com (S.-H.P.); ozkan.ozden@vanderbilt.edu (O.O.); \\ haiyan.jiang@vanderbilt.edu (H.J.); yong.cha@vanderbilt.edu (Y.I.C.); \\ daniel.pennington@vanderbilt.edu (J.D.P.); david.gius@vanderbilt.edu (D.G.) \\ 2 Free Radical and Radiation Biology Program, Department of Radiation Oncology, \\ Holden Comprehensive Cancer Center, The University of Iowa, Iowa City, IA 52242, USA; \\ E-Mails: naykinburns@uams.edu (N.A.-B.); douglas-spitz@uiowa.edu (D.R.S.)
}

* Author to whom correspondence should be addressed; E-Mail: hyun-seok.kim@vanderbilt.edu.

Received: 21 July 2011; in revised form: 14 September 2011 / Accepted: 20 September 2011 / Published: 23 September 2011

\begin{abstract}
One fundamental observation in cancer etiology is that the rate of malignancies in any mammalian population increases exponentially as a function of age, suggesting a mechanistic link between the cellular processes governing longevity and carcinogenesis. In addition, it is well established that aberrations in mitochondrial metabolism, as measured by increased reactive oxygen species (ROS), are observed in both aging and cancer. In this regard, genes that impact upon longevity have recently been characterized in $S$. cerevisiae and C. elegans, and the human homologs include the Sirtuin family of protein deacetylases. Interestingly, three of the seven sirtuin proteins are localized into the mitochondria suggesting a connection between the mitochondrial sirtuins, the free radical theory of aging, and carcinogenesis. Based on these results it has been hypothesized that Sirt3 functions as a mitochondrial fidelity protein whose function governs both aging and carcinogenesis by modulating ROS metabolism. Sirt3 has also now been identified as a genomically expressed, mitochondrial localized tumor suppressor and this review will outline potential relationships between mitochondrial ROS/superoxide levels, aging, and cell phenotypes permissive for estrogen and progesterone receptor positive breast carcinogenesis.
\end{abstract}


Keywords: Sirt3; mitochondria; acetylation; acetylome; cancer; MnSOD; carcinogenesis; receptor positive breast cancer

\section{Introduction}

Mammalian cells express proteins that protect against endogenous and exogenous forms of genotoxic stresses that induce genomic instability [1-3]. These proteins monitor the integrity of cellular metabolism as well as respond to stressful conditions by activating compensatory pathways [4-6]. An extension of this observation would be that the loss of function or genetic mutation of these fidelity proteins creates a cellular environment that is permissive for the development of tumors [7-10], suggesting that these proteins also function as tumor suppressor genes (TSG) in the context of aging and metabolism [11]. Since it is unlikely that evolutionary pressure selected for proteins in mammalian cells to prevent carcinogenesis, these proteins are more likely fidelity proteins that have evolved over time to protect specific organelles from damage induced by agents that induce genotoxic stress.

Genomic instability, a hallmark of cancer, is thought to be an early event in neoplastic transformation induced by genotoxic agents [12-14]. As such one of the paradigms in biology is that mammalian cells contain fidelity proteins that recognize DNA damage and subsequently initiate signaling cascades that maintain cellular processes to prevent the accumulation of genomic damage $[1,15,16]$. In this context the best known example of a tumor suppressor (TS) gene is p53, which senses cellular damage from multiple endogenous and exogenous genotoxic stresses, including ionizing radiation (IR), and induces reparative processes that prevent IR-induced genomic instability and carcinogenesis $[10,17,18]$. Thus, it has been proposed that the genomic instability contributes to cellular phenotypes that represent early events in the multi-step genetic, epigenetic, and metabolic processes contributing to carcinogenesis.

Mitochondria may also contain specific fidelity proteins that sense organelle conditions and activate signaling pathways to maintain mitochondrial homeostatic poise [19,20]. One of the primary functions of the mitochondria is the generation of ATP and complex aerobic organisms utilize oxygen as an electron acceptor in respiration, as an extremely efficient method of generating ATP [13]. However, by-products of mitochondrial electron transfer reactions in aerobic cells result in the production of ROS (i.e., superoxide and hydrogen peroxide) from the incomplete reduction of dioxygen [21]. The accumulation of abnormally high levels of mitochondrial superoxide from any source can create a condition referred to as "oxidative stress" that damages cells and is thought to contribute to both carcinogenesis and aging [22,23]. While low steady-state levels of ROS are easily tolerated and perform necessary signaling functions to coordinate metabolic and genetic processes [13], abnormally high levels of ROS from any number of possible sources can damage cells [24,25] and create a cellular environment permissive for genomic instability as well as carcinogenesis [2]. Thus, it has been proposed that mitochondrial damage, from any of a number of causes, might result in increased ROS that are causative agents in the development of genomic instability [26-28]. 


\section{Sirtuins as Fidelity or Tumor Suppressor Proteins}

Sirtuins are $\mathrm{NAD}^{+}$dependent class III histone deacetylases which are present from bacteria to humans $[29,30]$. Unlike traditional histone deacetylases, sirtuins dynamically deacetylate a variety of substrates ranging from transcription factors to metabolic enzymes as well as histones [31,32]. In addition, sirtuins require $\mathrm{NAD}^{+}$as a co-factor which makes them a metabolic sensor and connects their enzymatic activity to the energy and redox state of cells [33,34]. One theme that has emerged in the last several years is that aging, perhaps better defined as longevity, is a complex cellular process that appears to be regulated, at least in part, by a relatively new gene family referred to as sirtuins. Sirtuin genes are the human and murine homologs of the Saccharomyces cerevisiae Sir2 gene that has been shown to regulate both replicative and overall lifespan [3,4]. The sirtuin family genes also regulate longevity in C. elegans and D. melanogaster [35,36].

In lower organisms, sir2 plays a role in longevity by enhancing multiple mechanisms including but not limited to silencing of telomeres and sub-telomeric regions, silent mating type loci, and, crucially, the rDNA, suppressing formation of rDNA circles [36,37]. On the other hand, mammalian sirtuin members are associated with numerous physiological roles, such as stress response, regulation of metabolism, gene silencing, and aging [38,39]. While it has not been shown that these genes determine longevity in mammals they do appear to regulate critical signaling networks, and following stress, several mice lacking one of the sirtuin genes develop illnesses that mimic those observed in older humans $[36,40]$. Thus, it has been proposed that the mammalian sirtuins play a significant role, at least in part, in directing the acetylome signaling network that has recently been shown to be critical in the regulating multiple cellular processes [41]. These proteins share a common 275-amino acid catalytic domain and are localized to the nucleus (SIRT1, 6, and 7), mitochondria (SIRT3, 4, and 5), and cytoplasm (SIRT2), respectively [42]. Unlike histone deacetyl transferase the sirtuins primarily target cellular proteins other than histones suggesting these proteins are critical the regulation of cell signaling networks similar to phosphatases and kinases [39,41]. Sirtuins therefore appear to function as signaling proteins that post-translationally alter the activity of downstream protein targets via acetylation.

\section{Sirtuins Monitor and Direct Cellular Metabolism and Stress}

Sirtuin activity can be increased in response to metabolic, genotoxic, oxidative, and osmotic stresses. These stress responses appear to link to aging [43-45], oxidative stress, and the free-radical theory of aging (FRTA) [46]. In this model it is proposed that there is a mechanistic connection between aberrant cellular ROS levels and aging. Thus, it was proposed that an organism ages due to the unrepaired accumulation free radical damage to critical biomolecules as a function of time $[1,13]$. For most biological structures, free radical damage is closely associated with oxidative damage $[47,48]$. Thus, this theory would predict that antioxidants or reducing agents, may limit oxidative damage to biological structures by detoxifying free radicals and preventing aging and age-related human illness $[21,49,50]$.

Sirtuins, including the mitochondrial sirtuin Sirt3, appear to respond to changes in cellular and nutrient stress resulting in the activation of their deacetylase or ribosyltransferase activity resulting in a post-translational modification of downstream target proteins [20,50-53]. For example, it is now well established that Sirt3 deacetylation activity is activated by caloric restriction (CR) and fasting [20,50,53] 
and this induction in deacetylation activity also appears to protect against the development of age-related human pathology including cancer induction [20,54]. While the mice lacking Sirt3 do not exhibit changes in life span it has been shown in human studies that SIRT3 expression levels has been found to be associated with higher longevity [55]. In this regard, CR is the most effective non-genetic intervention described to increase lifespan across a wide variety of phyla [56]. While this phenomenon has been appreciated for many decades, the exact mechanism(s) remain unclear. While this effect has not been directly demonstrated in humans, humans may undergo many of the same metabolic changes and experience the same health effects in response to CR as seen in lower organisms [57]. In fact, it can be argued that $\mathrm{CR}$ may be the single best method identified to decrease the incidence of developing cancer and CR clearly decreases intracellular ROS [58]. Since it is now well established that CR activates sirtuins, including Sirt3, it seems reasonable to suggest a potential connection between sirtuins, cellular metabolism as measured by aberrant mitochondrial ROS, and age-related illness and pathologies including carcinogenesis.

\section{Sirtuins and Regulation of the Mitochondrial Acetylome}

The sirtuins appear to play a central role, at least in part, in regulating the cellular acetylome responding to endogenous and exogenous cell stress and nutrient distress [39,59]. Thus, is has been proposed that at least one function of the sirtuin gene family is the regulation and maintenance of the Metabolome via the deacetylation of specific downstream target proteins that direct intracellular oxidation/reduction pathways [37,41]. Reversible acetylation of lysine is a post-translational modification that neutralizes the positive charge of this amino acid, potentially altering the 3-dimensional structure of a protein as well as its enzymatic function [60,61]. One well known example of this is the histone deacetylases (HDACs) and histone acetyltransferases (HATs) that regulate gene expression through the modification of histone and changes in chromatin remodeling [62].

In this regard lysine acetylation has recently emerged as an important, and perhaps the primary, post-translational modification employed to regulate mitochondrial proteins $[63,64]$. Several proteomic surveys have identified a disproportionately high number of acetylated proteins in the mitochondria and many of these proteins are associated with energy homeostasis [64,65]. Reversible acetylation of lysines that alter protein structure is mediated by histone deacetylases (HDACs) and histone acetyltransferases (HATs) that regulate gene expression through the modification of histones [62]. Lysine acetylation is also involved in the regulation of p53 which appears to also play a role in mitochondrial redox regulation [66].

Sirt3 is the primary mitochondrial deacetylase and genetic knockout of Sirt3 alter mitochondrial protein acetylation [67] including aberrant/decreased mitochondrial ATP levels in vitro and in vivo [68]. Based on these results, acetylation of mitochondrial proteins may play a role in maintaining and regulating mitochondrial metabolism and function. Thus, we believe it is a logical extension to hypothesize that Sirt3 is a regulatory protein, maintaining mitochondrial homeostasis via changes in the acetylation of metabolic target proteins. This idea would mechanistically connect a metabolic sensing and/or signaling protein, such as Sirt3, to the direct regulation of mitochondrial energy metabolism, ATP synthesis, detoxification of mitochondrial ROS, as well as other biological processes essential for proper mitochondrial function. 


\section{Sirtuins, Mitochondrial ROS, and Carcinogenesis}

Since the rate of malignancies increases significantly as a function of age, several reports have suggested a potential mechanistic link between the cellular process governing longevity and the development of cancers [58,69]. In addition, it is well established that the mitochondria of tumor cells exhibit aberrant ROS and this observation has been suggested to account for the high degree of genomic instability demonstrated in cancer cells [46,70]. It is also a fundamental hypothesis in cancer research that increased or aberrant cellular mitochondrial ROS is an early event in cell damage that results in the generation of genomic stability that, under specific cellular conditions, can results in dedifferentiation and carcinogenesis $[12,13,71]$. These observations suggest a potential mechanistic connection between mitochondrial function and carcinogenesis; however, until recently there were a lack of genetic models to investigate this hypothesis.

Sirt3 appears to be the primary mitochondrial deacetylase it has been proposed that Sirt3 may function as a mitochondrial fidelity gene $[72,73]$. We hypothesized that critical regulators at the crossroads between aging and loss of Sirt3 deacetylase activity may create a cellular environment permissive for age-related cancers [19]. Thus, six years ago we, and others, constructed mice lacking the mitochondrial Sirt3 gene to determine if Sirt3 is a fidelity gene and if loss of function would create an in vivo tumor permissive phenotype.

Sirt3 is the mitochondrial matrix protein that is present in high amount in mouse kidney, heart, liver, and brown adipose tissues. Mice lacking Sirt3 do not display any significant observable acute physiological abnormalities; however, they have a large number of acetylated proteins in their mitochondria relative to wild-type mice [67,73]. However, when these animals or their cultured cells were challenged with various stress factors, such as oxidative stressors, chemical-hormonal, or ionizing radiation, they displayed physiological phenotypes consistent with increasing age. These phenotypes included cardiac hypertrophy [51,74], carcinogenesis [20,75,76], fatty liver [52,77], radiation-induced liver damage [50], and age-related hearing loss [54,78]. While it is unlikely that there is one all encompassing, overarching mechanism that underlie above-mentioned in vivo phenotypes, each of these publications identified increased cellular ROS as a potential underlying etiology and suggest a potential connection between the development of these pathologies and aberrant mitochondrial metabolism. Therefore these results support the hypothesis that excessive levels of ROS is one mechanism of shortened life span and age-associated pathological conditions, as suggested by the free radical theory of aging $[46,70]$.

\section{SIRT3 Is a Genomically Expressed, Mitochondrial Localized TS Protein}

In this regard, Sirt3 was reported to protect cells against ROS by enhancing the activity of antioxidant defense system, suggesting that this protein could be a crucial mitochondrial fidelity protein in response to oxidative stress $[20,50,53,54]$. Whereas Sirt3 deficiency was well tolerated in young mice in normal conditions, at ages greater than one year old Sirt3 knockout mice exhibited a deterioration liver mitochondrial DNA, lower ATP production, increase mitochondrial ROS including superoxide levels, and developed ER/PR positive breast malignancies [20]. These results suggested that Sirt3 might function as a mitochondrial fidelity protein and loss of function of Sirt3 may establish cellular environment permissive for aberrant mitochondrial metabolism, oxidative stress, and carcinogenesis. 
In this regard, Sirt3 knockout mouse embryonic fibroblasts (MEFs) exposed to various stress factors, such as oxidative, genotoxic stress, and radiation exposures resulted in a loss of contact inhibition. The Sirt ${ }^{-/-}$MEFs were also immortalized and transformed by infection with a single oncogene suggesting that Sirt3 might function as an in vitro tumor suppressor. These results were confirmed when mice lacking Sirt3 spontaneously developed mammary tumors appearing at just over one year of age. Finally, the interrogation of human breast cancer samples as measured via: (1) staining for protein levels; (2) RT-PCR for RNA levels; and (3) genomic analysis all showed a statistically significant decrease in SIRT3 levels in tumor cells, as compared to normal tissue controls. As such, we would propose that these results very strongly suggest that SIRT3 is a genomically expressed, mitochondrially localized tumor suppressor protein [20]. In this regard, the Sirt3 knockout mice do not begin to develop mammary tumors until after 12 months of age the immunohistochemical staining has identified these tumors as well differentiated estrogen (ER) and progesterone (PR) receptor positive mammary tumors [20]. This result seems logical since these mice lack a gene that has been suggested to play a role in aging or, perhaps better stated, anti-aging, and receptor positive breast malignancies are most common in post-menopausal women and have a very strong statistically correlation with increasing age [58,69]. In fact breast cancer has a very specific age-related incidence that increases slowly until the mid 60's when a significant increase in incidence is observed [58].

While our work [20], and another rigorously done manuscript [75,76], have both shown that Sirt3 functions as a TS, the detailed mechanism linking Sirt3-mediated mitochondrial ROS and carcinogenesis still remains to be fully determined. In this regard, the subcellular localization of Sirt3 is in close proximity to the main source of ROS production and this renders Sirt3 in the ideal location for the protection from aging and age-associated disorders including carcinogenesis. Elevated mitochondrial oxidative stress was proposed to play a role, at least in part, for the development of breast cancers in humans $[79,80]$ as well as the development of mammary tumors in the aged Sirt3 knockout mice. Consistent with this data, human breast tissue samples also displayed increased mitochondrial superoxide levels and decreased Sirt3 expression and the Sirt $3^{-/-}$mouse hepatocytes [20] and cardiomyoctes $[51,74]$ also showed significantly higher basal superoxide levels that were observed to further increase when exposed to different types of exogenous cellular stress challenges. These results suggested, but did not prove, a direct connection between aberrant mitochondrial superoxide levels and the tumor permissive phenotype observed in mice and MEFs lacking Sirt3.

A more convincing argument for the role of aberrant mitochondrial superoxide levels in cells lacking Sirt3 was shown using duel infection experiments with lentivirus expressing MnSOD. MnSOD is the primary superoxide detoxification enzyme that converts superoxide to hydrogen peroxide that is finally converted to water by catalase [81]. In these tissue culture experiments, co-infection of lentiMnSOD not only decreased mitochondrial superoxide levels but also prevented immortalization of $\mathrm{Sirt}^{-/-}$MEFs by a single oncogene [20]. These experiments were confirmed using a MnSOD gene where lysine 122 was mutated to an arginine, $\mathrm{MnSOD}^{122 \mathrm{~K}-\mathrm{R}}$, resulting in a constitutively active dominant positive MnSOD protein [50]. Co-infection of lenti-MnSOD ${ }^{122 \mathrm{~K}-\mathrm{R}}$ also prevented immortalization of $\mathrm{Sirt}^{-/-}$MEFs by a single oncogene. In contrast, co-infection with mutated dominant negative MnSOD gene, lenti-MnSOD ${ }^{122 \mathrm{~K}-\mathrm{Q}}$, mimicking a constitutively acetylated lysine, failed to prevent immortalization by infection with a single oncogene [50]. Similar experiments have shown that MnSOD lysine 68 [82] and lysines 53 and 89 [53] also appear to be deacetylated by SIRT3 as well as alter MnSOD enzymatic 
activity. Finally, it has also shown that infection of a MnSOD dominant positive (lenti-MnSOD ${ }^{122 \mathrm{~K}-\mathrm{R}}$ ) gene prevented tissue culture transformation with exogenous agents such as ionizing radiation as well as stress-induced increases in cellular ROS [50]. All these experiments strongly suggest that aberrant mitochondrial superoxide metabolism, at least in part, plays a significant role in the tumor permissive phenotype observed in cells lacking Sirt3.

While this work, as well as that recently published from the Guarente and Haigis laboratories [75,76,83], suggests a mechanistic connection, at least in part, between aberrant mitochondrial superoxide metabolism and carcinogenesis it seems like factors other, such as HIF-1 $\alpha$ than metabolism are involved. For example, we now know that a variety of important metabolic and cell survival related mitochondrial targets of Sirt3 including acetyl-CoA synthetase [63,84], glutamate dehydrogenase (GDH) [84,85], long-chain acyl-CoA dehydrogenase (LCAD) [46], succinate dehydrogenase [86] and mitochondrial ribosome subunit MRPL10 [87]. Sirt3 has also be shown to have pro-apoptotic or anti-apoptotic effects on different cell types and at least on mechanism involves deacetylating $\mathrm{Ku} 70$ and consequently preventing the release of BAX into mitochondria [88]. Finally, several manuscripts have identified multiple mitochondrial proteins that contain proteins (Figure 1) with reversible acetyl lysine(s) and many of these have been shown to be altered in cancers $[64,65,67]$. Thus, similar to $\mathrm{p} 53$, aberrant regulation of several proteins in the mitochondrial acetylome may play some role in the cellular damage and tumor permissive phenotype observed in mice lacking Sirt3.

Figure 1. Proposed model describing Sirt3 acetylation and subsequent regulation of downstream target proteins enzymatic activity. Sirt3 is localized into the inner mitochondrial membrane and appears to be activated by agents that induce oxidative stress and respond to aberrant or increased mitochondrial levels of superoxide. Sirt3 has been shown to regulate the activity of other mitochondrial proteins including acetyl-CoA synthetase [63,84], glutamate dehydrogenase (GDH) [84,85], long-chain acyl-CoA dehydrogenase (LCAD) [46], succinate dehydrogenase [86,87], and mitochondrial ribosome subunit MRPL10 $[87,88]$. Sirt3 has also be shown to have pro-apoptotic or anti-apoptotic effects on different cell types and at least on mechanism involves deacetylating $\mathrm{Ku} 70$ and consequently preventing the release of BAX into mitochondria $[88,89]$.

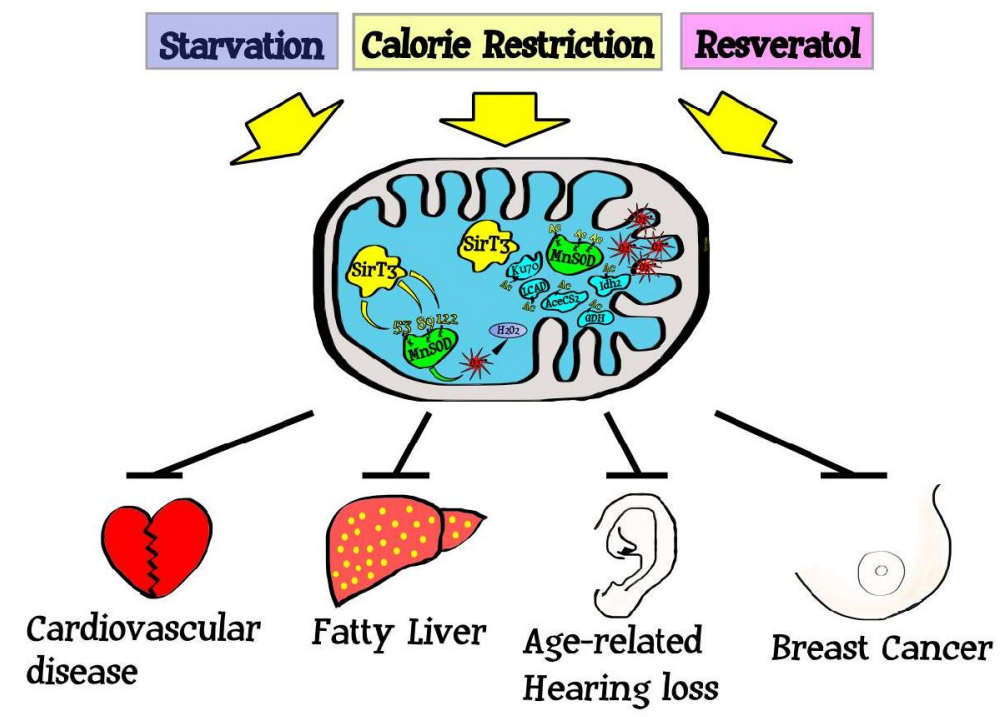


One final question is how aberrant regulation of the mitochondrial acetylome, results in spontaneous, stress-induced genomic instability and can loss of proteins other than Sir3 result in spontaneous genomic instability [1]. In this regard, a somewhat similar finding was recently reported in Saccharomyces cerevisiae by the Gottschling laboratory [90]. In this work it was shown that a reduction in the mitochondrial membrane potential resulted in a cellular crisis identified via a defect in iron-sulfur cluster (ISC) biogenesis, which is required for normal mitochondrial function. This crisis resulted in downregulation of ISC protein biogenesis causing genomic instability, suggesting mitochondrial dysfunction stimulates nuclear genome instability by inhibiting the production of ISC-containing proteins.

Our results also suggest that increased mitochondrial superoxide levels, which exceed the ability of MnSOD to detoxify superoxide, may escape the mitochondria and subsequently increase both the cytoplasmic and nuclear superoxide levels. However, this idea is controversial since many researchers believe that superoxide cannot move out of the mitochondria. Thus, can superoxide generated in mitochondria enter the cytosol via passage through voltage dependent or independent channels in the mitochondrial membranes causing alterations in redox signaling in the cytosol, potentially contributing to genomic instability in the nucleus? The evidence supporting this hypothesis is mostly indirect using anion channel blockers such as DIDS (4,4'-diisothiocyanatostilbene-2,2'-disulfonate) [91,92] or other methods [93]. While this review cannot answer this controversy, at least one mechanism for the increased genomic instability in mice and MEFs lacking Sirt3 appears to involve increased mitochondrial superoxide levels that use either existing ISC or other membrane channels to escape into cellular organelles outside of the mitochondria.

\section{Conclusions}

One fundamental observation in eukaryotic biology is that mammalian cells contain fidelity proteins that function to monitor the integrity of critical intracellular processes, and deletion or mutation of the corresponding genes results in a cellular environment permissive for the accumulation of damage $[10,11,94]$. Thus, it seems like a logical extension that the mitochondria would also contain fidelity proteins to maintain the integrity of the mitochondria. In this regard, loss of Sirt3 results in a series of stress and/or aging-related murine phenotypes including receptor positive breast cancer [20], fatty liver [52,77], insulin resistance [52], cardiac hypertrophy [51,74], radiation-induced liver steatosis [50], and age-associated hearing loss [54]. While it may be premature to make a cause and effect connection between age-related pathologies and alteration in mitochondrial ROS it seems likely that aberrant mitochondrial ROS plays a role, at least in part, in these murine phenotypes. Finally, it also seems clear that Sirt3 is a mitochondrial localized tumor suppressor or fidelity protein and identifying the downstream deacetylation targets of Sirt3 will lead to better understanding of mechanism linking mitochondrial fidelity, carcinogenesis, and aging.

\section{Acknowledgments}

DG is supported by 1R01CA152601-01 and 1R01CA152799-01A1 from the NCI, BC093803 from the DOD, and SPORE P50CA98131. DRS and NAB were supported by grants from the NIH and DOE (R01CA133114, P30CA086862, and DE-SC0000830). 


\section{References}

1. Slane, B.G.; Aykin-Burns, N.; Smith, B.J.; Kalen, A.L.; Goswami, P.C.; Domann, F.E.; Spitz, D.R. Mutation of succinate dehydrogenase subunit $\mathrm{C}$ results in increased $\mathrm{O} 2$.- , oxidative stress, and genomic instability. Cancer Res. 2006, 66, 7615-7620.

2. Aykin-Burns, N.; Ahmad, I.M.; Zhu, Y.; Oberley, L.W.; Spitz, D.R. Increased levels of superoxide and $\mathrm{H} 2 \mathrm{O} 2$ mediate the differential susceptibility of cancer cells versus normal cells to glucose deprivation. Biochem. J. 2009, 418, 29-37.

3. Du, C.; Gao, Z.; Venkatesha, V.A.; Kalen, A.L.; Chaudhuri, L.; Spitz, D.R.; Cullen, J.J.; Oberley, L.W.; Goswami, P.C. Mitochondrial ROS and radiation induced transformation in mouse embryonic fibroblasts. Cancer Biol. Ther. 2009, 8, 1962-1971.

4. Mallakin, A.; Sugiyama, T.; Taneja, P.; Matise, L.A.; Frazier, D.P.; Choudhary, M.; Hawkins, G.A.; D'Agostino, R.B., Jr.; Willingham, M.C.; Inoue, K. Mutually Exclusive Inactivation of DMP1 and ARF/p53 in Lung Cancer. Cancer Cell 2007, 12, 381-394.

5. Uren, A.G.; Kool, J.; Matentzoglu, K.; de Ridder, J.; Mattison, J.; van Uitert, M.; Lagcher, W.; Sie, D.; Tanger, E.; Cox, T.; et al. Large-scale mutagenesis in p19ARF-and p53-deficient mice identifies cancer genes and their collaborative networks. Cell 2008, 133, 727-741.

6. Vousden, K.H.; Prives, C. Blinded by the Light: The Growing Complexity of p53. Cell 2009, 137, 413-431.

7. Greger, V.; Passarge, E.; Hopping, W.; Messmer, E.; Horsthemke, B. Epigenetic changes may contribute to the formation and spontaneous regression of retinoblastoma. Hum. Genet. 1989, 83, $155-158$.

8. Baker, S.J.; Markowitz, S.; Fearon, E.R.; Willson, J.K.; Vogelstein, B. Suppression of human colorectal carcinoma cell growth by wild-type p53. Science 1990, 249, 912-915.

9. Feinberg, A.P.; Johnson, L.A.; Law, D.J.; Kuehn, S.E.; Steenman, M.; Williams, B.R.; Thomas, G.; Boland, C.R.; Rainier, S.; Koi, M. Multiple tumor suppressor genes in multistep carcinogenesis. Tohoku. J. Exp. Med. 1992, 168, 149-152.

10. Sherr, C.J.; McCormick, F. The Rb and p53 pathways in cancer. Cancer Cell 2002, 2, 103-112.

11. Hunter, T. Oncoprotein networks. Cell 1997, 88, 333-346.

12. Clutton, S.M.; Townsend, K.M.; Walker, C.; Ansell, J.D.; Wright, E.G. Radiation induced genomic instability and persisting oxidative stress in primary bone marrow cultures. Carcinogenesis 1996, 17, 1633-1639.

13. Spitz, D.R.; Azzam, E.I.; Li, J.J.; Gius, D. Metabolic oxidation/reduction reactions and cellular responses to ionizing radiation: a unifying concept in stress response biology. Cancer Metastasis Rev. 2004, 23, 311-322.

14. Duesberg, P. Does aneuploidy or mutation start cancer? Science 2005, 307, 41.

15. Deng, C.X. BRCA 1 : Cell cycle checkpoint, genetic instability, DNA damage response and cancer evolution. Nucleic Acids Res. 2006, 34, 1416-1426.

16. Wang, R.H.; Sengupta, K.; Li, C.; Kim, H.S.; Cao, L.; Xiao, C.; Kim, S.; Xu, X.; Zheng, Y.; Chilton, B.; et al. Impaired DNA damage response, genome instability, and tumorigenesis in SIRT1 mutant mice. Cancer Cell 2008, 14, 312-323. 
17. Parada, L.F.; Land, H.; Weinberg, R.A.; Wolf, D.; Rotter, V. Cooperation between gene encoding p53 tumour antigen and ras in cellular transformation. Nature 1984, 312, 649-651.

18. Taylor, W.R.; Egan, S.E.; Mowat, M.; Greenberg, A.H.; Wright, J.A. Evidence for synergistic interactions between ras, myc and a mutant form of p53 in cellular transformation and tumor dissemination. Oncogene 1992, 7, 1383-1390.

19. Saunders, L.R.; Verdin, E. Sirtuins: critical regulators at the crossroads between cancer and aging. Oncogene 2007, 26, 5489-5504.

20. Kim, H.S.; Patel, K.; Muldoon-Jacobs, K.; Bisht, K.S.; Aykin-Burns, N.; Pennington, J.D.; van der Meer, R.; Nguyen, P.; Savage, J.; Owens, K.M.; et al. SIRT3 is a mitochondria-localized tumor suppressor required for maintenance of mitochondrial integrity and metabolism during stress. Cancer Cell 2010, 17, 41-52.

21. Gius, D.; Botero, A.; Shah, S.; Curry, H.A. Intracellular oxidation/reduction status in the regulation of transcription factors NF-[kappa] B and AP-1. Toxicol. Lett. 1999, 106, 93-106.

22. Pelicano, H.; Carney, D.; Huang, P. ROS stress in cancer cells and therapeutic implications. Drug Resist. Updat. 2004, 7, 97-110.

23. Valko, M.; Izakovic, M.; Mazur, M.; Rhodes, C.J.; Telser, J. Role of oxygen radicals in DNA damage and cancer incidence. Mol. Cell. Biochem. 2004, 266, 37-56.

24. Spitz, D.R.; Li, G.C. Heat-induced cytotoxicity in $\mathrm{H}_{2} \mathrm{O}_{2}$-resistant Chinese hamster fibroblasts. J. Cell Physiol. 1990, 142, 255-260.

25. Sies, H. Role of reactive oxygen species in biological processes. Klin. Wochenschr. 1991, 69, 965-968.

26. Singh, K.K. Mitochondria damage checkpoint, aging, and cancer. Ann. NY Acad. Sci. 2006, 1067, 182-190.

27. Valko, M.; Rhodes, C.J.; Moncol, J.; Izakovic, M.; Mazur, M. Free radicals, metals and antioxidants in oxidative stress-induced cancer. Chem. Biol. Interact. 2006, 160, 1-40.

28. Chen, J.; Kadlubar, F.F.; Chen, J.Z. DNA supercoiling suppresses real-time PCR: a new approach to the quantification of mitochondrial DNA damage and repair. Nucleic Acids Res. 2007, 35, 1377-1388.

29. Tanny, J.C.; Dowd, G.J.; Huang, J.; Hilz, H.; Moazed, D. An Enzymatic Activity in the Yeast Sir2 Protein that Is Essential for Gene Silencing. Cell 1999, 99, 735-745.

30. Kaeberlein, M.; McVey, M.; Guarente, L. The SIR2/3/4 complex and SIR2 alone promote longevity in Saccharomyces cerevisiae by two different mechanisms. Genes Dev. 1999, 13, 2570-2580.

31. Imai, S.; Armstrong, C.M.; Kaeberlein, M.; Guarente, L. Transcriptional silencing and longevity protein Sir2 is an NAD-dependent histone deacetylase. Nature 2000, 403, 795-800.

32. Landry, J.; Sutton, A.; Tafrov, S.T.; Heller, R.C.; Stebbins, J.; Pillus, L.; Sternglanz, R. The silencing protein SIR2 and its homologs are NAD-dependent protein deacetylases. Proc. Natl. Acad. Sci. USA 2000, 97, 5807-5811.

33. Lin, S.J.; Defossez, P.A.; Guarente, L. Requirement of NAD and SIR2 for life-span extension by calorie restriction in Saccharomyces cerevisiae. Science 2000, 289, 2126-2128.

34. North, B.J.; Verdin, E. Sirtuins: Sir2-related NAD-dependent protein deacetylases. Genome Biol. 2004, 5, 224. 
35. Rogina, B.; Helfand, S.L. Sir2 mediates longevity in the fly through a pathway related to calorie restriction. Proc. Natl. Acad. Sci. USA 2004, 101, 15998-16003.

36. Wood, J.G.; Rogina, B.; Lavu, S.; Howitz, K.; Helfand, S.L.; Tatar, M.; Sinclair, D. Sirtuin activators mimic caloric restriction and delay ageing in metazoans. Nature 2004, 430, 686-689.

37. Guarente, L.; Partridge, L.; Wallace, D.C. Molecular Biology of Aging; Cold Spring Harbor Laboratory Press: Woodbury, NY, USA, 2008.

38. Haigis, M.C.; Guarente, L.P. Mammalian sirtuins-emerging roles in physiology, aging, and calorie restriction. Genes Dev. 2006, 20, 2913-2921.

39. Finkel, T.; Deng, C.X.; Mostoslavsky, R. Recent progress in the biology and physiology of sirtuins. Nature 2009, 460, 587-591.

40. Kim, E.J.; Kho, J.H.; Kang, M.R.; Um, S.J. Active regulator of SIRT1 cooperates with SIRT1 and facilitates suppression of p53 activity. Mol. Cell 2007, 28, 277-290.

41. Smith, K.T.; Workman, J.L. Introducing the acetylome. Nat. Biotechnol. 2009, 27, 917-919.

42. Hallows, W.C.; Albaugh, B.N.; Denu, J.M. Where in the cell is SIRT3?-Functional localization of an NAD+-dependent protein deacetylase. J. Biochem.2008, 411, e11-e13.

43. Chua, K.F.; Mostoslavsky, R.; Lombard, D.B.; Pang, W.W.; Saito, S.; Franco, S.; Kaushal, D.; Cheng, H.L.; Fischer, M.R.; Stokes, N.; et al. Mammalian SIRT1 limits replicative life span in response to chronic genotoxic stress. Cell Metab. 2005, 2, 67-76.

44. Droge, W.; Schipper, H.M. Oxidative stress and aberrant signaling in aging and cognitive decline. Aging Cell 2007, 6, 361-370.

45. Scher, M.B.; Vaquero, A.; Reinberg, D. SirT3 is a nuclear NAD+-dependent histone deacetylase that translocates to the mitochondria upon cellular stress. Genes Dev. 2007, 21, 920-928.

46. Harman, D. Aging: a theory based on free radical and radiation chemistry. J. Gerontol. 1956, 11, 298-300.

47. Valko, M.; Leibfritz, D.; Moncol, J.; Cronin, M.T.; Mazur, M.; Telser, J. Free radicals and antioxidant in normal physiological function and human disease. Int. J. Biochem. Cell Biol. 2007, $39,44-84$.

48. Buffenstein, R.; Edrey, Y.H.; Yang, T.; Mele, J. The oxidative stress theory of aging: embattled or invincible? Insights from non-traditional model organisms. Age (Dordr) 2008, 30, 99-109.

49. Diamond, D.A.; Parsian, A.; Hunt, C.R.; Lofgren, S.; Spitz, D.R.; Goswami, P.C.; Gius, D. Redox factor-1 (Ref-1) mediates the activation of AP-1 in HeLa and NIH 3T3 cells in response to heat shock. J. Biol. Chem. 1999, 274, 16959-16964.

50. Tao, R.; Coleman, M.C.; Pennington, J.D.; Ozden, O.; Park, S.H.; Jiang, H.; Kim, H.S.; Flynn, C.R.; Hill, S.; Hayes McDonald, W.; et al. Sirt3-mediated deacetylation of evolutionarily conserved lysine 122 regulates MnSOD activity in response to stress. Mol. Cell 40, 893-904.

51. Sundaresan, N.R.; Samant, S.A.; Pillai, V.B.; Rajamohan, S.B.; Gupta, M.P. SIRT3 is a stressresponsive deacetylase in cardiomyocytes that protects cells from stress-mediated cell death by deacetylation of Ku70. Mol. Cell Biol. 2008, 24, 6384-6401.

52. Hirschey, M.D.; Shimazu, T.; Goetzman, E.; Jing, E.; Schwer, B.; Lombard, D.B.; Grueter, C.A.; Harris, C.; Biddinger, S.; Ilkayeva, O.R.; et al. SIRT3 regulates fatty acid oxidation via reversible enzyme deacetylation. Nature 2010, 464, 121-125. 
53. Qiu, X.; Brown, K.; Hirschey, M.D.; Verdin, E.; Chen, D. Calorie restriction reduces oxidative stress by SIRT3-mediated SOD2 activation. Cell Metab. 2010, 12, 662-667.

54. Someya, S.; Yu, W.; Hallows, W.C.; Xu, J.; Vann, J.M.; Leeuwenburgh, C.; Tanokura, M.; Denu, J.M.; Prolla, T.A. Sirt3 mediates reduction of oxidative damage and prevention of age-related hearing loss under caloric restriction. Cell 2010, 143, 802-812.

55. Lescai, F.; Blanche, H.; Nebel, A.; Beekman, M.; Sahbatou, M.; Flachsbart, F.; Slagboom, E.; Schreiber, S.; Sorbi, S.; Passarino, G.; Franceschi, C. Human longevity and 11p15. 5: A study in 1321 centenarians. Eur. J. Hum. Genet. 2009, 17, 1515-1519.

56. Hursting, S.D.; Lavigne, J.A.; Berrigan, D.; Perkins, S.N.; Barrett, J.C. Calorie Restriction, Aging, and Cancer Prevention: Mechanisms of Action and Applicability to Humans*. Annu. Rev. Med. 2003, 54, 131-152.

57. Sinclair, D.A. Toward a unified theory of caloric restriction and longevity regulation. Mech. Ageing Dev. 2005, 126, 987-1002.

58. Ershler, W.B.; Longo, D.L. Aging and cancer: issues of basic and clinical science J. Natl. Cancer Inst. 1997, 89, 1489-1497.

59. Nemoto, S.; Fergusson, M.M.; Finkel, T. Nutrient availability regulates SIRT1 through a forkhead-dependent pathway. Science 2004, 306, 2105-2108.

60. Kouzarides, T. Chromatin Modifications and Their Function. Cell 2007, 128, 693-705.

61. Yang, X.J. Lysine acetylation and the bromodomain: a new partnership for signaling. Bioessays 2004, 26, 1076-1087.

62. Shahbazian, M.D.; Grunstein, M. of site-specific histone acetylation and deacetylation. Annu. Rev. Biochem. 2007, 76, 75-100.

63. Schwer, B.; Bunkenborg, J.; Verdin, R.O.; Andersen, J.S.; Verdin, E. Reversible lysine acetylation controls the activity of the mitochondrial enzyme acetyl-CoA synthetase 2. Proc. Natl. Acad. Sci. USA 2006, 103, 10224-10229.

64. Choudhary, C.; Kumar, C.; Gnad, F.; Nielsen, M.L.; Rehman, M.; Walther, T.C.; Olsen, J.V.; Mann, M. Lysine acetylation targets protein complexes and co-regulates major cellular functions. Science 2009, 325, 834-840.

65. Kim, S.C.; Sprung, R.; Chen, Y.; Xu, Y.; Ball, H.; Pei, J.; Cheng, T.; Kho, Y.; Xiao, H.; Xiao, L.; et al. Substrate and functional diversity of lysine acetylation revealed by a proteomics survey. Mol. Cell 2006, 23, 607-618.

66. Yang, H.; Baur, J.A.; Chen, A.; Miller, C.; Adams, J.K.; Kisielewski, A.; Howitz, K.T.; Zipkin, R.E.; Sinclair, D.A. Design and synthesis of compounds that extend yeast replicative lifespan. Aging Cell 2007, 6, 35-43.

67. Lombard, D.B.; Alt, F.W.; Cheng, H.L.; Bunkenborg, J.; Streeper, R.S.; Mostoslavsky, R.; Kim, J.; Yancopoulos, G.; Valenzuela, D.; Murphy, A.; et al. Mammalian Sir2 homolog SIRT3 regulates global mitochondrial lysine acetylation. Mol. Cell Biol. 2007, 27, 8807-8814.

68. Ahn, B.H.; Kim, H.S.; Song, S.; Lee, I.H.; Liu, J.; Vassilopoulos, A.; Deng, C.X.; Finkel, T. A role for the mitochondrial deacetylase Sirt3 in regulating energy homeostasis. Proc. Natl. Acad. Sci. USA 2008, 105, 14447-14452.

69. Ershler, W.B.; Longo, D.L. The biology of aging. Cancer 1997, 80, 1284-1293. 
70. Gemma, C.; Vila, J.; Bachstetter, A.; Bickford, P.C. Early inhibition of TNF [alpha] increases 6-hydroxydopamine-induced striatal degeneration. Brain Res. 2007, 1147, 240-247.

71. Dayal, D.; Martin, S.M.; Limoli, C.L.; Spitz, D.R. Hydrogen peroxide mediates the radiationinduced mutator phenotype in mammalian cells. Biochem. J. 2008, 413, 185-191.

72. Schwer, B.; Eckersdorff, M.; Li, Y.; Silva, J.C.; Fermin, D.; Kurtev, M.V.; Giallourakis, C.; Comb, M.J.; Alt, F.W.; Lombard, D.B. Calorie restriction alters mitochondrial protein acetylation. Aging Cell 2009, 8, 604-606.

73. Lombard, D.B. Sirtuins at the breaking point: SIRT6 in DNA repair. Aging (Albany NY) 2009, 1, $12-16$.

74. Sundaresan, N.R.; Gupta, M.; Kim, G.; Rajamohan, S.B.; Isbatan, A.; Gupta, M.P. Sirt3 blocks the cardiac hypertrophic response by augmenting Foxo3a-dependent antioxidant defense mechanisms in mice. J. Clin. Invest. 2009, 119, 2758-2771.

75. Bell, E.L.; Emerling, B.M.; Ricoult, S.J.; Guarente, L. SirT3 suppresses hypoxia inducible factor $1 \alpha$ and tumor growth by inhibiting mitochondrial ROS production. Oncogene 2011, 30, 2986-2996.

76. Finley, L.W.; Carracedo, A.; Lee, J.; Souza, A.; Egia, A.; Zhang, J.; Teruya-Feldstein, J.; Moreira, P.I.; Cardoso, S.M.; Clish, C.B.; Pandolfi, P.P.; Haigis, M.C. SIRT3 Opposes Reprogramming of Cancer Cell Metabolism through HIF1 a Destabilization. Cancer Cell 2011, 19, 416-428.

77. Kendrick, A.A.; Choudhury, M.; Rahman, S.M.; McCurdy, C.E.; Friederich, M.; Vanhove, J.L.; Watson, P.A.; Birdsey, N.; Bao, J.; Gius, D.; et al. Fatty liver is associated with reduced SIRT3 activity and mitochondrial protein hyperacetylation. Biochem. J. 2011, 433, 505-514.

78. Sebastian, C.; Mostoslavsky, R. SIRT3 in Calorie Restriction: Can You Hear Me Now? Cell 2010, 143, 667-668.

79. Deng, Y.T.; Huang, H.C.; Lin, J.K. Rotenone induces apoptosis in MCF-7 human breast cancer cell-mediated ROS through JNK and p38 signaling. Mol. Carcinog. 2010, 49, 141-151.

80. Pujana, M.A.; Han, J.D.; Starita, L.M.; Stevens, K.N.; Tewari, M.; Ahn, J.S.; Rennert, G.; Moreno, V.; Kirchhoff, T.; Gold, B.; et al. Network modeling links breast cancer susceptibility and centrosome dysfunction. Nat. Genet. 2007, 39, 1338-1349.

81. Oberley, L.W.; Oberley, T.D. Role of antioxidant enzymes in cell immortalization and transformation. Mol. Cell Biochem. 1988, 84, 147-153.

82. Chen, Y.; Zhang, J.; Lin, Y.; Lei, Q.; Guan, K.L.; Zhao, S.; Xiong, Y. Tumour suppressor SIRT3 deacetylates and activates manganese superoxide dismutase to scavenge ROS. EMBO Rep. 12, 534-541.

83. Schumacker, P.T. SIRT3 Controls Cancer Metabolic Reprogramming by Regulating ROS and HIF. Cancer Cell 2011, 19, 299-300.

84. Hallows, W.C.; Lee, S.; Denu, J.M. Sirtuins deacetylate and activate mammalian acetyl-CoA synthetases. Proc. Natl. Acad. Sci. USA 2006, 103, 10230-10235.

85. Schlicker, C.; Gertz, M.; Papatheodorou, P.; Kachholz, B.; Becker, C.F.; Steegborn, C. Substrates and regulation mechanisms for the human mitochondrial sirtuins Sirt3 and Sirt5. J. Mol. Biol. 2008, 382, 790-801.

86. Cimen, H.; Han, M.J.; Yang, Y.; Tong, Q.; Koc, H.; Koc, E.C. Regulation of succinate dehydrogenase activity by SIRT3 in mammalian mitochondria. Biochemistry 2010, 49, 304-311. 
87. Yang, Y.; Cimen, H.; Han, M.J.; Shi, T.; Deng, J.H.; Koc, H.; Palacios, O.M.; Montier, L.; Bai, Y.; Tong, Q.; Koc, E.C. NAD+-dependent deacetylase SIRT3 regulates mitochondrial protein synthesis by deacetylation of the ribosomal protein MRPL10. J. Biol. Chem. 2010, 285, 7417-7429.

88. Marfe, G.; Tafani, M.; Indelicato, M.; Sinibaldi-Salimei, P.; Reali, V.; Pucci, B.; Fini, M.; Russo, M.A. Kaempferol induces apoptosis in two different cell lines via Akt inactivation, Bax and SIRT3 activation, and mitochondrial dysfunction. J. Cell Biochem. 2009, 106, 643-650.

89. Allison, S.J.; Milner, J. SIRT3 is pro-apoptotic and participates in distinct basal apoptotic pathways. Cell Cycle 2007, 6, 2669-2677.

90. Veatch, J.R.; McMurray, M.A.; Nelson, Z.W.; Gottschling, D.E. Mitochondrial dysfunction leads to nuclear genome instability via an iron-sulfur cluster defect. Cell 2009, 137, 1247-1258.

91. Chandel, N.S.; McClintock, D.S.; Feliciano, C.E.; Wood, T.M.; Melendez, J.A.; Rodriguez, A.M.; Schumacker, P.T. Reactive oxygen species generated at mitochondrial complex III stabilize hypoxia-inducible factor-1 during hypoxia. A mechanism of $\mathrm{O}_{2}$ sensing. J. Biol. Chem. 2000, 275, 25130-25138.

92. Han, D.; Antunes, F.; Canali, R.; Rettori, D.; Cadenas, E. Voltage-dependent anion channels control the release of the superoxide anion from mitochondria to cytosol. J. Biol. Chem. 2003, 278, 5557-5563.

93. Kulisz, A.; Chen, N.; Chandel, N.S.; Shao, Z.; Schumacker, P.T. Mitochondrial ROS initiate phosphorylation of p38 MAP kinase during hypoxia in cardiomyocytes. Am. J. Physiol. Lung Cell Mol. Physiol. 2002, 282, L1324-L1329.

94. Sherr, C.J. Principles of tumor suppression. Cell 2004, 116, 235-246.

(C) 2011 by the authors; licensee MDPI, Basel, Switzerland. This article is an open access article distributed under the terms and conditions of the Creative Commons Attribution license (http://creativecommons.org/licenses/by/3.0/). 